\title{
CORTISONE IN OPHTHALMOLOGY
}

\author{
By A. J. B. Goldsmith, F.R.C.S. and H. E. HobBs, F.R.C.S.
}

With its complexity of tissues and clear media through which the reactions of many of these tissues can be directly observed under considerable magnification, the eye is in many ways the ideal experimental organ in which to observe the effects of a powerful agent such as cortisone; it is not surprising, therefore, that since the introduction of this drug a considerable mass of experimental and clinical observation has accumulated from which generally accepted indications for, and methods of, treatment have been derived.

In any consideration of the proper place of cortisone in ocular therapeutics, particularly when it is administered, as it generally is, by local application either by drops or by subconjunctival injection, it is essential always to bear in mind its local clinical effects. These, in ocular disease may be summarized, in so far as is possible at the present time, by saying that it blocks temporarily the exudative phases of inflammation, whether bacterial, allergic or traumatic in origin; that by inhibiting fibroblast formation in the process of repair it reduces the severity of, or in favourable circumstance may even prevent the damage and and scarring which may be catastrophic visually, inherent in the later processes of resolution, fibrosis and repair generally; while-most important of all from the long-term therapeutic point of viewit influences, in no way that one is aware of, the basic cause of the disease process. It has been confirmed by clinical experience that it is entirely without effect on the organized sequelae of organic disease, nor has it any effect on degenerative conditions except in so far as they, or some of them, may, during some period of their evolution, be associated with exudative phenomena.

It follows from this very brief summary of its broad clinical effects that cortisone might be expected to be of greatest value in the treatment of certain acute inflammatory diseases of the eye, and in practice this has proved to be the case. In the management of such diseases it is fair to say that cortisone has provided the greatest advance in ocular therapeutics since the introduction of the various chemotherapeutic and antibiotic drugs now at our disposal. At first sight this statement may appear anomalous since it has been expressly stated that cortisone does not influence the funda- $\vec{\omega}$ mental cause of the disease process, but two points must be borne in mind. The first is that manyo inflammatory diseases of the eye are self-limiting, either in their entirety or in their relapsing acute $\stackrel{+}{+}$ exacerbations; the second, that in the eye a very ${ }_{\tilde{c}}$ small area of scarring, which in practically every other tissue of the body would be without demon- $\vec{\omega}$ strable effect on its economy and function, may have serious or disastrous results so far as vision? is concerned, as, for example, corneal scars result $-\overrightarrow{-}$ ing from various forms of keratitis, blocking up of the pupil by organization of the exudate pouredô out in a case of acute iritis and damage to the macular region of the retina by the oedema, gre later by the scarring engendered by a disturbante in an adjacent region of the choroid. It is in inhibition of such concomitants and sequelae of the acute inflammatory process that the great valueo of cortisone lies, so far as ocular diseases are con-0ू cerned; but in using it, spectacular though itsळ results may be, it is essential always to remember that in many of the conditions in which it is used $B$ the underlying causes of disease, the causes which? may well be responsible for subsequent relapses? remain unaffected, and all possible steps must be taken to eliminate these if a cure in the real sense 3 . of the term is to be achieved. It cannot be too' strongly emphasized that the making of a red eye white, gratifying though this may be, does not mean that everything necessary in the manage 3 ment of the case has been accomplished.

Before discussing some conditions in which cortisone is of proven value its mode of administra을. tion may be briefly reviewed. The effect of thon drug upon the particular ocular lesion will, of of course, depend upon the local concentration achieved and, where this is in the superficiato tissues - conjunctiva, episclera or superficial corneab layers-drops of a dilution of $I: 5$ or $I: 10$ of the standard suspension (2.5 or $5 \mathrm{mg}$. per ml.) ar£ usually effective. As a maintenance dose they may? be used two or three times a day while in acuteo cases they may be given hourly. In acute inflam? mations of the anterior segment-iridocyclitis 


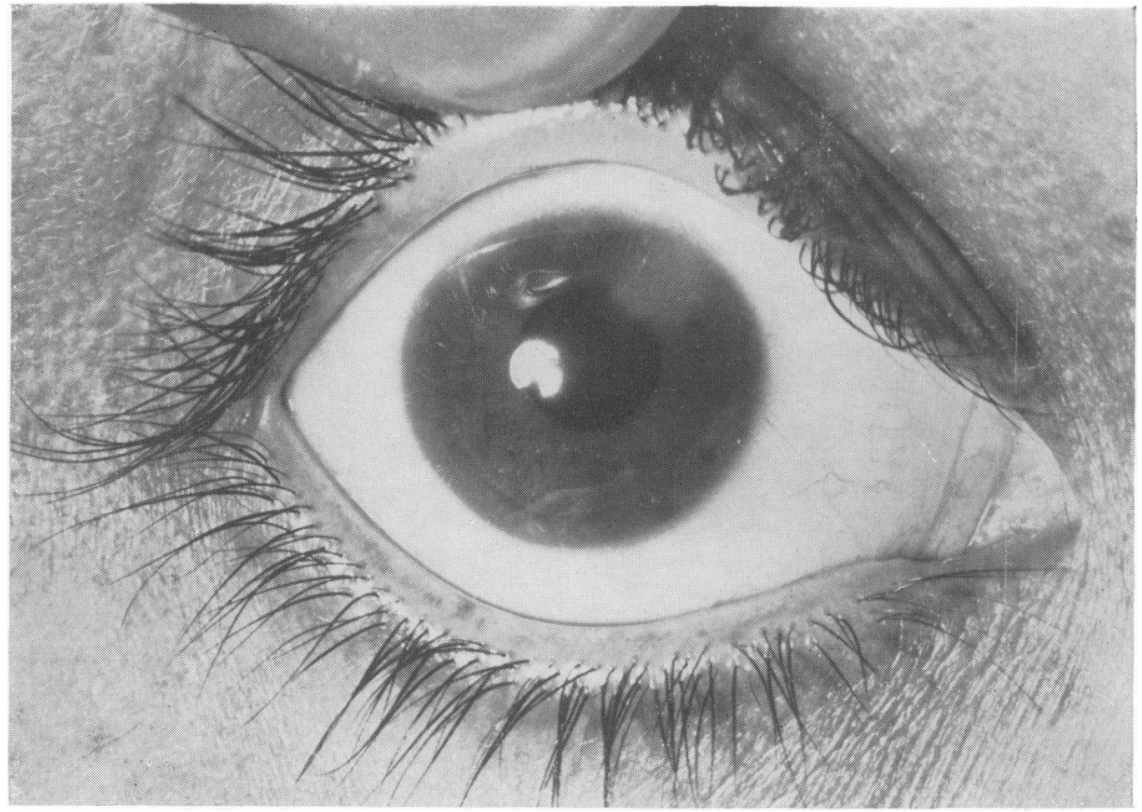

FIg. I.-Syphilitis interstitial keratitis. Local cortisone treatment at onset of the attack. Eventual visual acuity 6/6. Faint corneal scars only.

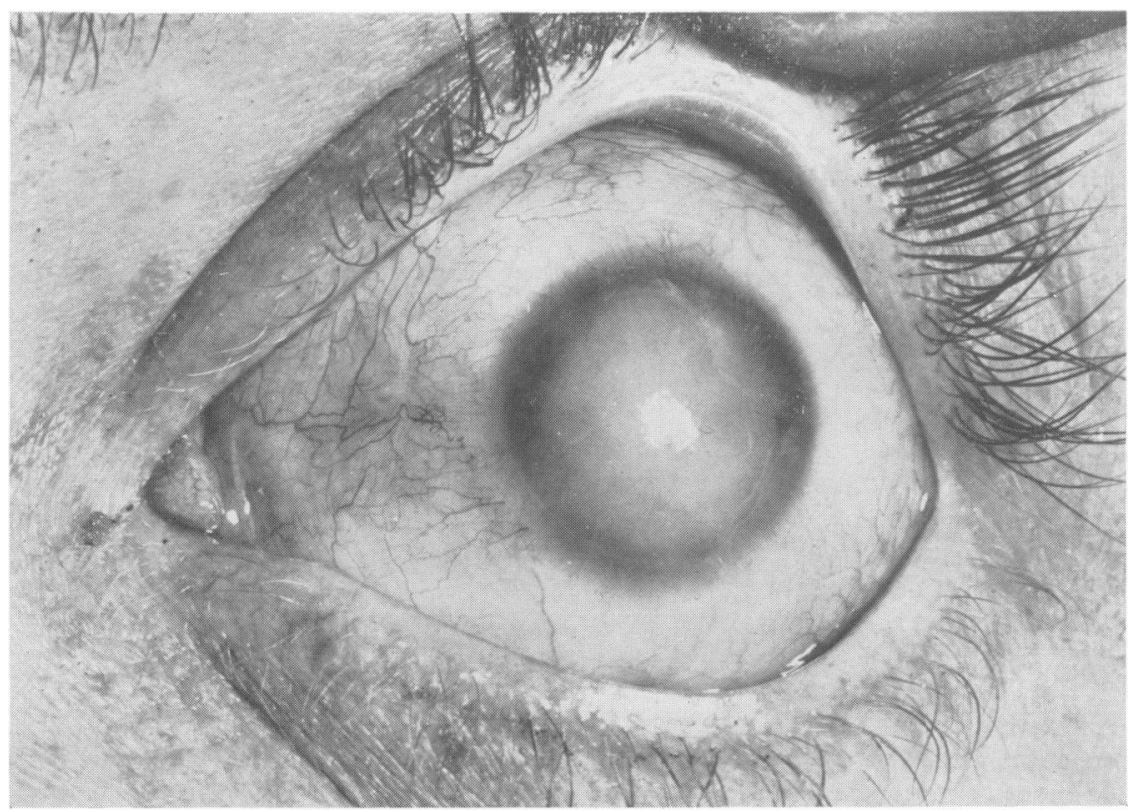

FIG. 2.- Syphilitis interstitial keratitis. In this case cortisone was started only after a period of systemic anti-syphilitic treatment and when the keratitis was in a florid state. The end result is a dense central corneal scar and reduction of visual acuity to hand movements. 
interstitial keratitis, etc. $-0.25 \mathrm{ml}$. (6.25 mg.) of the standard suspension is often given by injection under the conjunctiva; whilst, if the posterior segment of the globe is affected, the injection may be made further back, into Tenon's capsule. The injections, which cause little or no reaction, may be repeated every three or four days if necessary or may be superseded by drops once the desired effect is achieved. Fortunately, in such cases, the dosage is so small that no risk of side effects need be considered and no precautions in regard to such need be taken.

It is, of course, usual in cases treated with cortisone, however well they may be expected to respond, to continue with such other local treatment-atropine, antibiotics and the like-as may be indicated, while as soon as possible steps should be taken to investigate or, when known, to treat any systemic condition which may have a bearing on the local disease.

Certain conditions, to which brief reference will be made later, are also treated by systemic cortisone and, of course, ACTH; in these the mode of administration, dosage and precautions follow general principles which need not be discussed; during such treatment, however, the eye should be kept under the most careful observation since its response is not always that anticipated and desired.

Whether local or systemic administration is used, a word of warning is called for in those cases diagnosed as of tuberculous aetiology. In these, if the local reaction is allergic, proliferative and exudative, in nature, immediate benefit may well accrue; if, however, the local reaction is caseating (and the differentiation may well be clinically impossible) the use of either cortisone or ACTH may lead to an almost immediate deterioration in the condition of the eye, an incication of course for the immediate cessation of these drugs and a change to the appropriate antibiotic therapy.

In the ensuing very brief discussion of illustrative specific ocular diseases in which cortisone is used more emphasis will be laid on those in which it is of proven value than on the others in which its effect is merely symptomatic or capricious. Of the former, the first to be mentioned is interstitial keratitis. In its common form this is a manifestation of congenital syphilis occurring usually in late childhood or adolescence. Both eyes tend to be affected, often with an interval which may be of months or even years between the two, but it is unusual to have more than one attack in each eye. Systemic antisyphilitic treatment, if given during the course of an attack in one eye, has no influence on its evolution; nor, given at this stage or earlier, does it diminish the liability of the second or of both eyes as the case may be, to be affected by the disease. Clinically the affected eye is inflamed red and painful, while there is a diffuse haze of the cornea which is soon followed by the growth into. it of superficial and deep blood vessels. Patho logically there is, in the early stages, an inflam $\stackrel{?}{+}$ matory oedema of the deep layers of the corne? with separation of the lamellae, a heavy leucocytie infiltration and ingrowth of new vessels from the limbal region. As the inflammatory process sub@ sides, there is left more or less dense scarring ow the deeper part of the cornea with permanent vascularization and opacity which is usually dense enough to interfere fairly seriously with cleat vision, while the presence of blood vessels pres judices the chances of a subsequent successfu. keratoplasty in that sooner or later the graft itselp. may become invaded by these vessels and becomes opaque.

In these cases, if cortisone is administered at an early stage, the obvious signs of inflammation, th pain and redness of the eye disappear, the cornea clears remarkably rapidly, its neovascularization is inhibited or, if it has already started, ceases to $\sigma$ progress and the later sequelae of scarring anc opacification are prevented. The comparison be tween a case treated in the early stage with cor tisone and one first treated only when the corfes was heavily vascularized, is well seen in illustrations (Figs. I and 2). It is interesting thap although the response to cortisone may be quite dramatic and the eye may look almost normas within a few days, the use of the drug must bE continued for some six to eight weeks, which is the length of time the attack would have persisted in more or less florid form had its active manifesta $\frac{0}{3}$ tions not been masked in this manner; if it is discontinued during this period the obvious disease processes become reactivated. What pres cisely is happening in the corneal tissues durings this time to suppress their response to the noxious: factor causing the inflammation is not known? From the fact that the other cornea may later be involved it would appear, however, that the process is a purely local one.

The aetiology of many cases of acute iridocyclitis remains speculative and even in those cases in which a definite association with diseases else where, such as Still's disease, ankylosing spondy litis and posterior urethritis, can clearly be demon+O strated, treatment of the apparently primary condition cannot with certainty prevent recurrents ocular trouble. This, however, is no reason fo not taking such measures as may be possible to reduce the frequency and severity of the attack $\$$ even although the ravages of these may be completely controlled. In iridocyclitis cortisone is of most value in those attacks associated with marked exudation, and in such its use even for a few days 
only helps very greatly in controlling secondary glaucoma, in preventing the formation of adhesions of the iris to the lens and in the angle of the anterior chamber, and in preventing organization of the exudate to form a membrane occluding the pupil and interfering thereby with vision. In the more chronic, granulomatous forms of iridocyclitis, in which plastic exudation is not a prominent feature, cortisone is correspondingly of far less value and is perhaps best used only to tide over a temporary crisis until other therapeutic measures can begin to take effect. Somewhat similar considerations apply in cases of acute choroiditis affecting the posterior part of the uveal tract, although the response of acute choroiditis to cortisone given either systemically or by orbital injection is capricious and is by no means as marked, nor as satisfactory, as that of acute inflammations of the anterior segment of the eye.

The beneficial effects seen in iridocyclitis may present dramatically in cases of sympathetic ophthalmitis. In this condition, which typically follows a perforating wound of the ciliary region of one eye, a severe generalized inflammation of the. whole uveal tract (iris, ciliary body and choroid) and retina develops, affecting not only the injured eye, but also the previously normal eye and progresses only too frequently to disruption of the whole ocular economy, shrinking of the eyes and complete blindness, the whole unfortunate course being accompanied by severe physical pain as well as the mental stress obviously involved. The pathogenesis of the condition is still obscure but it appears most likely on the evidence available to be primarily a virus infection of the uveal tract, probably with a secondary development of allergy to uveal pigment which tends to keep up activity once the primary virus infestation has died out. The possibility of sympathetic ophthalmitis has always been a grave anxiety to surgeons dealing with wounds of the eye and with these the generally accepted policy has always been to consider seriously excision of the eye, even when potentially useful, if the more active signs of inflammation do not show marked and definite evidence of subsiding within 10 to 14 days. With the advent of cortisone, however, this policy has shown a change, and, provided that the inflammation responds to the use of cortisone and that there is a reasonable chance of the injured eye being of value functionally or even only cosmetically, most surgeons feel happier in making more prolonged attempts to conserve the injured eye without feeling that they are running an unjustifiable risk in regard to both. Fortunately, also, even in established cases of sympathetic ophthalmitis, cortisone may be as useful as in interstitial keratitis, although in the former disease its use may have to be continued for as long as two years and when it is discontinued the eye must be watched carefully so that it can be resumed if necessary. There is no clinical indication as to when eyes suffering from this condition will be safe once the protective influence of cortisone is withdrawn.

As a corollary it will be obvious that cortisone will be of equal value in such conditions as operative wounds, in which, although the danger of sympathetic ophthalmitis is not anticipated, the eye reacts unfavourably either inherently, through the trauma involved, or by the liberation of irritant material such as soft lens matter. In these cases cortisone in the small quantities used seems to have little or no prejudicial effect on wound healing and often helps to tide over the early post-operative period and prevents more serious complications developing such as, for example, the invasion of the graft by blood vessels in an eye which has recently been subjected to the operation of keratoplasty.

In many acute localized lesions of the cornea, sclera and conjunctiva cortisone has a valuable role to play, provided always that adequate measures are taken to determine the cause and treat it where it is detectable. In infective conditions, provided that the infection has been brought under control, cortisone is unlikely adversely to affecto healing when employed topically in the usual amounts. Intractable conjunctivitis, spring catarrh, phlyctenular kerato-conjunctivitis, episcleritis and: rosacea keratitis are all conditions in which its value appears to be chiefly in producing symptomatic relief and administration may have to be prolonged; in all of them it would seem justifiable to use cortisone in default or sometimes in anticipation of response to other therapeutic measures; but in view of our ignorance of the effects-if any-of really prolonged administration, it would equally seem advisable to take such other measures as may be available to achieve permanent cure or even only quasi-permanent symptomatic relief.

Finally there may be mentioned certain conditions in the treatment of which systemic cortisone or ACTH appears to offer a more efficient means of controlling the ocular manifestations of the disease than has been hitherto available.

Opacification of the vitreous by the passage into it of inflammatory exudates is an inconstant but grave concomitant of uveitis, either as iridocyclitis or as posterior uveitis, and the prognosis for ultimate visual recovery when once dense vitreous opacities have formed becomes progressively worse the longer their absorption is delayed. Cortisone, if it can reach the vitreous in adequate concentration during the stage of active exudation, may produce rapid clearing of the vitreous and dramatic visual improvement and in such cases the supple- 
menting of intratenon injections by systemic ACTH is valuable.

In cranial arteritis an ocular concomitant is blindness, usually bilateral, due to involvement not of the central retinal artery but presumably of the small nutrient twigs supplying the optic nerve and usually occurring some little time after the other manifestations of pain in the face and head and tenderness of the temporal vessels. Immediate symptomatic relief of the latter group of symptoms is usually given by systemic cortisone in small doses; it is not known in what proportion of cases treated or untreated by cortisone, blindness supervenes, but the symptomatic relief, and the possibility that it may avert the ocular complication make its use eminently justifiable, especially as there is no other known method of preventing the lesion of the optic nerve, nor of alleviating it and restoring vision once it has developed.

Similar considerations apply in some of the demyelinating lesions, particularly perhaps neuromyslitis optica (Devic's disease), a comparatively rare condition characterized ocularly by a bilateral optic neuritis. This is of varying severity and it is impossible to predict the outcome in terms of permanent visual impairment. Systemic cortisone in the early stages, however, often effects considerable immediate visual improvement, but it is impossible at the present time to say whether or not this is reflected in the eventual visual results.

To summarize, cortisone cannot be said to cure any ocular disease and its action is confined to the time it is administered and shortly thereafter. Its use can in no way justifiably replace specific aetiological investigation and treatment, since in the majority of the diseases in which it is used the ultimate prognosis is dominated by the tendency to relapses. Within this limitation its value is very great, particularly in acute cases and when the cause of the malady is eradicable, or the inflammation is self-limiting. There is no value, and every possible danger, in its haphazard administration in every chronic and recalcitrant disease affecting the eye particularly if, in these cases, it is allowed to become a substitute for rational consideration, diagnosis, investigation and treatment of the particular case.

\title{
CHEMOTHERAPEUTIC AND ANTIBIOTIC DRUGS
}

\author{
Reprinted from the Postgraduate Medical Journal
}

Price: 5s. the set, plus 3d. postage

INTRODUCTION: THE PRINCIPLES OF CHEMOTHERAPY

C. A. Keele, M.D., F.R.C.P.

CHEMOTHERAPY OF NON-TUBERCULOUS

DISEASES OF THE CHEST

Horace Joules, M.D., F.R.C.P.

CHEMOTHERAPY OF PULMONARY

TUBERCULOSIS

J. G. Scadding, M.D., F.R.C.P.

CHEMOTHERAPY OF NON-PULMONARY

TUBERCULOUS CONDITIONS

Peter H. Buxton, M.R.C.P.

CHEMOTHERAPY AND THE VENEREAL

DISEASES

R. R. Willcox, M.D.
CHEMOTHERAPY OF TROPICAL INFECTIONS OF THE BOWEL

A. R. D. Adams, M.D., F.R.C.P., D.T,M.

CHEMOTHERAPY OF MALARIA

Prof. F. Murgatroyd, M.D., F.R.C.P., D.T.M.

CHEMOTHERAPY OF TUBERCULOUS INFECTIONS OF THE URINARY TRACT Arthur Jacobs, F.R.F.P.S.

CHEMOTHERAPY OF NON-TUBERCULOUS INFECTIONS OF THE URINARY TRACT

F. R. Kilpatrick, M.S., F.R.C.S., and Kenneth Maclean, M.D., M.R.C.P.

MOST RECENT ANTIBIOTIC AGENTS

G. Brownlee, Ph.D., D.Sc.

Published by

THE FELLOWSHIP OF POSTGRADUATE MEDICINE

60, Portland Place, London, W.1 